\title{
Scalable Exploration of Functional Dependency by Interpolation and Incremental SAT Solving
}

\author{
Chih-Chun Lee, Jie-Hong R. Jiang, Chung-Yang (Ric) Huang \\ EE Dept./Graduate Inst. of Electronics Engineering \\ National Taiwan University \\ Taipei 10617, Taiwan
}

\author{
Alan Mishchenko \\ Dept. of EECS \\ University of California, Berkeley \\ CA94720, USA
}

\begin{abstract}
Functional dependency is concerned with rewriting a Boolean function $f$ as a function $h$ over a set of base functions $\left\{g_{1}, \ldots, g_{n}\right\}$, i.e. $f=h\left(g_{1}, \ldots, g_{n}\right)$. It plays an important role in many aspects of electronic design automation (EDA), ranging from logic synthesis to formal verification. Prior approaches to the exploration of functional dependency are based on binary decision diagrams (BDDs), which may not be easily scalable to large designs. This paper proposes a novel reformulation that extensively exploits the capability of modern satisfiability (SAT) solvers. Thereby, functional dependency is detected effectively through incremental SAT solving, and the dependency function $h$, if it exists, is obtained through Craig interpolation. The main strengths of the proposed approach include: (1) fast detection of functional dependency with modest memory consumption and thus scalable to large designs, (2) a full capacity to handle a large set of base functions and thus discovering dependency whenever exists, and (3) potential application to large-scale logic optimization and verification reduction. Experimental results show the proposed method is far superior to prior work and scales well in dealing with the largest ISCAS89 and ITC99 benchmark circuits with up to $200 \mathrm{~K}$ gates.
\end{abstract}

\section{INTRODUCTION}

Functional dependency [1] appears commonly among a set of Boolean functions $\left\{f_{1}, \ldots, f_{n}\right\}$ in VLSI circuit design as a function $f_{i}$ (called the target function) can often be reexpressed as some function $h$ (called the dependency function) over a subset of the functions (called the base functions). The exploration of functional dependency plays an important role in many aspects of EDA, ranging from logic synthesis to formal verification. For instance, it leads to the identification of redundant registers in RTL synthesis [2][3], resubstitution and simplification of Boolean functions in both technology-independent and technologydependent logic synthesis [4], BDD minimization [5] and state space reduction [1][6][7] in formal verification, search space reduction in SAT solving [8], etc. Advances on the exploration of functional dependency may benefit a wide range of applications.

Given a set of Boolean functions $\left\{f_{1}, \ldots, f_{n}\right\}$, we would like to know if any $f_{i}$ can be written as $h\left(f_{1}, \ldots, f_{i-1}, f_{i+1}, \ldots, f_{n}\right)$. Conventional approaches [1] to the exploration of functional dependency rely mostly on BDDs [9]. Unfortunately computation using BDDs suffers from the memory explosion problem and thus is not scalable to manipulate large designs. In contrast, SAT solving consumes little memory (linear in the input size) at the cost of time resources and thus is more robust at least in representing large designs. Recent advances, see e.g. [10][11], in SAT solving have made it a very efficient Boolean reasoning engine and a viable alternative to BDD. More and more logic synthesis and verification algorithms shift their computation paradigm from BDD to SAT, e.g. [12][13]. However, formulating the computation of functional dependency as pure SAT solving is not straightforward due to the difficulty in deriving the dependency function $h$, whose derivation in BDD-based computation is in contrast immediate.

This paper demonstrates, for the first time, that the exploration of functional dependency (including efficient derivation of dependency function) can be achieved with pure SAT solving. In particular, a dependency function, if it exists, can be obtained through the construction of interpolants from a refutation proof of a SAT solver. Essentially, the Craig interpolation theorem [14] lays the foundation. Moreover, to detect functional dependency for different target functions and to obtain different dependency functions for a target function, incremental SAT solving is adopted to reuse learned clauses and to indicate conflict assumptions. Even though incremental SAT solving is not new and has been widely used, we explore its new use in our framework. In essence, it not only speeds up the computation, but also provides an automatic way of selecting sets of base functions for a target function.

The main results of the paper include (1) a new SAT-based derivation of dependency function using Craig interpolation, which enables a pure SAT solution to the exploration of functional dependency, and (2) an incremental SAT-based enumeration of target and base functions, which provides an automatic way of identifying useful base functions and effectively reduces the search space for solving similar SAT instances. Experiments show encouraging improvements over BDD-based approaches. As the pure SAT-based computation of functional dependency avoids the BDD memory explosion problem, it is scalable to large designs and turns out to be powerful in detecting functional dependency even among a large set of base functions.

The paper is organized as follows. After preliminaries are introduced in Section 2, our SAT formulation of functional dependency is detailed in Section 3. The proposed approach is evaluated with experimental results in Section 4. Section 5 concludes this paper and outlines some future research directions.

\section{PRELIMINARIES}

As a notational convention, in the sequel symbols " $\wedge$ ", “ $\vee$ ", and " $\neg$ " denote Boolean AND, OR, and COMPLEMENT operations, respectively. The cardinality (or size) of a set $\mathrm{S}$ is denoted as $|\mathrm{S}|$. The problem formulation of functional dependency and some background on SAT solving are given as follows.

\subsection{Functional Dependency}

Functional dependency is defined as follows. 
Definition 1. Given a Boolean function $f: \boldsymbol{B}^{m} \rightarrow \boldsymbol{B}$ and a vector of Boolean functions $G=\left(g_{1}(X), \ldots, g_{n}(X)\right)$ with $g_{i}: \boldsymbol{B}^{m} \rightarrow \boldsymbol{B}$ for $i=$ $1, \ldots, n$, over the same set of variable vector $X=\left(x_{1}, \ldots, x_{m}\right)$, we say that $f$ functionally depends on $G$ if there exists a Boolean function $h: \boldsymbol{B}^{n} \rightarrow \boldsymbol{B}$, called the dependency function, such that $f(X)=h\left(g_{1}(X), \ldots, g_{n}(X)\right)$. We call functions $f$, $G$, and $h$ the target function, base functions, and dependency function, respectively.

Note that functions $f$ and $G$ are over the same domain in the definition; $h$ needs not depend on all of the functions in $G$.

The necessary and sufficient condition of the existence of the dependency function $h$ is given as follows.

Proposition 1. [1] Given a target function $f$ and base functions $G$, let $h^{0}=\left\{a \in \boldsymbol{B}^{n}: a=G(b)\right.$ and $\left.f(b)=0, b \in \boldsymbol{B}^{m}\right\}$ and $h^{1}=\left\{a \in \boldsymbol{B}^{n}\right.$ : $a=G(b)$ and $\left.f(b)=1, b \in \boldsymbol{B}^{m}\right\}$. Then $h$ is a feasible dependency function if and only if $\left\{h^{0} \cap h^{1}\right\}$ is empty. In this case, $h^{0}, h^{1}$, and $\boldsymbol{B}^{n} \backslash\left\{h^{0} \cup h^{1}\right\}$ are the off-set, on-set, and don't-care set of $h$, respectively.

By Proposition 1, one can not only determine the existence of a dependency function, but also deduce a feasible one.

To explore functional dependency for a given circuit netlist, there are many choices of $f$ and $G$. One may ask how to effectively choose $G$ for a specific $f$.

Definition 2. For a Boolean function $f$ with input variables $X=$ $\left(x_{1}, \ldots, x_{m}\right)$, variable $x_{i}$ is a support variable of $f$ if $f\left(x_{1}, \ldots, x_{i-1}, 0\right.$, $\left.x_{i+1}, \ldots, x_{m}\right) \neq f\left(x_{1}, \ldots, x_{i-1}, 1, x_{i+1}, \ldots, x_{m}\right)$.

For a functional vector $G=\left(g_{1}, g_{2}, \ldots, g_{n}\right)$, its support variables are the union of the support variables of $g_{i}$ for $i=1, \ldots, n$.

\subsubsection{BDD-based Exploration of Functional}

Dependency

Conventional BDD-based exploration of functional dependency is reviewed in order to contrast with the novel SAT-based approach.

Proposition 1 suggests a way of determining the existence of a dependency function and its derivation. Essentially standard image computation applies. Let $y_{i}$ be the output variable of $g_{i}$. Then the on-set, off-set, and dc-set of $h$ can be derived by

$$
\begin{aligned}
& h^{0}(Y)=\exists X[R(X, Y) \wedge(f(X) \equiv 0)], \\
& h^{1}(Y)=\exists X[R(X, Y) \wedge(f(X) \equiv 1)], \text { and } \\
& h^{\mathrm{dc}}(Y)=\neg\left(h^{0} \vee h^{1}\right),
\end{aligned}
$$

respectively, where relation $R(X, Y)=\left(y_{1} \equiv g_{1}(X)\right) \wedge\left(y_{2} \equiv g_{2}(X)\right) \wedge$ $\cdots \wedge\left(y_{n} \equiv g_{n}(X)\right)$. The dependency function $h(Y)$ exists if and only if $\left(h^{0}(Y) \wedge h^{1}(Y)\right) \equiv 0$. All of the above operations can be done using BDDs; see also [4].

Note that constructing the relation $R(X, Y)$ along with the image computation may suffer from memory explosion especially when $|G|$ is large even though the final BDDs of $h^{0}$ and $h^{1}$ can be small. Therefore, it is necessary to restrict the size of the set of base functions at the cost of losing completeness. Keeping $|G|$ small may often result in a failure to compute some dependency that truly holds in a circuit. Once the search for a feasible dependency function with respect to a set of base functions fails, another set of base functions is selected and the computation of functional dependency repeats. Consequently, although some fast filtering techniques, e.g. by Proposition 2, are available [1], BDDbased computation is inefficient in that there may be too many selections of $G$ tested before functional dependency is discovered.
As will be seen later, the deficiency can be overcome in SATbased exploration of functional dependency.

\subsection{Propositional Satisfiability}

Let $V=\left\{v_{1}, \ldots, v_{k}\right\}$ be a finite set of Boolean variables. A literal $l$ is either a Boolean variable $v_{i}$ or its negated form $\neg v_{i}$. A clause $c$ is a disjunction of literals. Without loss of generality, we shall assume there are no repeated or complementary literals in the same clause. A SAT instance is a conjunction of clauses, i.e., in the so-called conjunctive normal form (CNF). In the sequel, a clause set $C=\left\{c_{1}, \ldots, c_{k}\right\}$ shall mean to be the $\operatorname{CNF}\left(c_{1} \wedge \cdots \wedge c_{k}\right)$. An assignment over $V$ gives every variable $v_{i}$ a Boolean value either true or false. A SAT instance is satisfiable if there exists a satisfying assignment such that the CNF evaluates to true. Otherwise it is unsatisfiable. Given a SAT instance, the satisfiability (SAT) problem asks whether it is satisfiable or not. A SAT solver is designated to solve the SAT problem.

Definition 3. Assume literal $v$ is in clause $c_{1}$ and $\neg v$ in $c_{2}$. A resolution of clauses $c_{1}$ and $c_{2}$ on variable $v$ yields a new clause $c$ containing all literals in $c_{1}$ and $c_{2}$ except for $v$ and $\neg v$. The clause $c$ is called the resolvent of $c_{1}$ and $c_{2}$, and variable $v$ the pivot variable.

Proposition 3. A resolvent $c$ of $c_{1}$ and $c_{2}$ is a logical consequence of $c_{1} \wedge c_{2}$, that is, $c_{1} \wedge c_{2}$ implies $c$.

Theorem 1. [15] For an unsatisfiable SAT instance, there exists a sequence of resolution steps leading to an empty clause.

Theorem 1 can be easily proved by Proposition 3 since an unsatisfiable SAT instance must imply a contradiction. Often only a subset of the clauses of the SAT instance participates in the resolution steps leading to an empty clause.

Definition 4. $A$ refutation proof $\Pi$ of an unsatisfiable $S A T$ instance $C$ is a directed acyclic graph (DAG) $\Gamma=(N, A)$, where every node in $N$ represents a clause which is either a root clause in $C$ or a resolvent clause having exactly two predecessor nodes, and every arc in A connects a node to its ancestor node. The unique leaf of $\Pi$ corresponds the empty clause.

Modern SAT solvers, such as Chaff [10] and MiniSat [11], are capable of producing a refutation proof from an unsatisfiable SAT instance.

\subsubsection{Refutation Proof and Craig's Interpolation}

Theorem 2. (Craig Interpolation Theorem) [14] Given two inconsistent clause sets $A$ and $B$ (i.e. the clause set $A \cup B$ is unsatisfiable), then there exists a Boolean formula $A^{\#}$ referring only to the common input variables of $A$ and $B$ such that $A \Rightarrow A^{\#}$ and $A^{\#} \Rightarrow \neg B$.

The Boolean formula $A^{\#}$ is referred to as the interpolant of $A$ and $B$. Detailed exposition on how to construct an interpolant from a refutation proof in linear time can be found in [16][17][18]. Note that the so-derived interpolant is in a circuit structure, which can then be converted into $\mathrm{CNF}$ as discussed below.

\subsubsection{Circuit to CNF Conversion}

Given a circuit netlist, it can be converted to a CNF in such a way that the satisfiability is preserved. The conversion is achievable in linear time by introducing some intermediate variables [19][20]. 


\section{SAT-BASED EXPLORATION OF FUNCTIONAL DEPENDENCY}

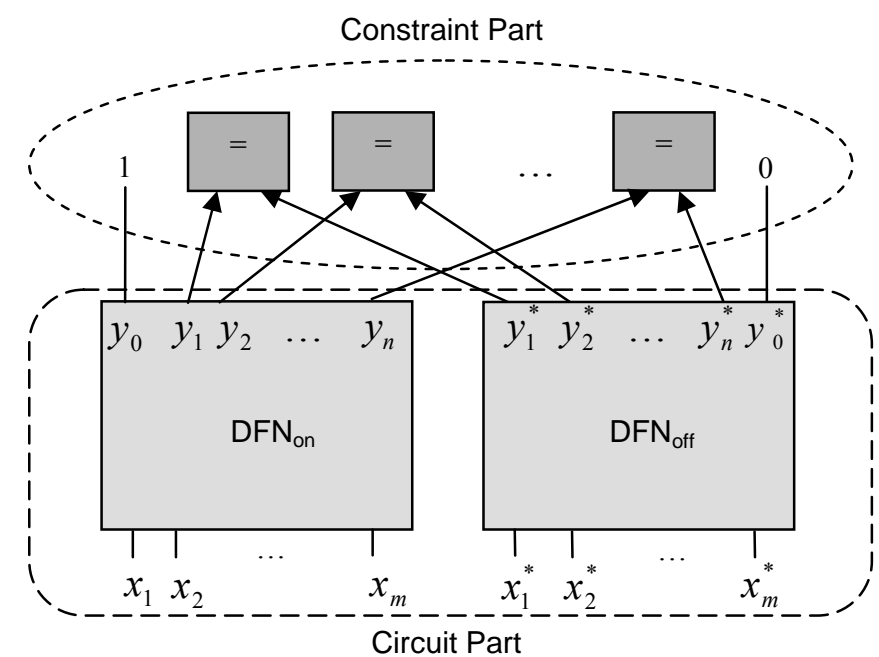

Figure 1. Dependency Function Network.

\subsection{The Primary Construct}

To formulate the exploration of functional dependency as SAT solving, we introduce the dependency function network (DFN) as shown in Fig. 1. For a given circuit netlist consisting of $n+1$ Boolean functions $\left\{f_{0}, \ldots, f_{n}\right\}$, suppose function $f_{0}$ and the others are identified to be the target function and base functions, respectively. That is, in the notation of Section $2, f_{0}$ corresponds to $f$ and $f_{i}$ corresponds to $g_{i}$ for $i=1, \ldots, n$. The circuit netlist is instantiated into two copies, identified as $\mathrm{DFN}_{\text {on }}$ and $\mathrm{DFN}_{\text {off }}$, in the DFN. For every variable $v$ (respectively function $f$ ) in $\mathrm{DFN}_{\mathrm{on}}$, there is starred counterpart $v^{*}$ (respectively $\left.f^{*}\right)$ in $\mathrm{DFN}_{\text {off. Let }} y_{i}$ and $y_{i}{ }^{*}$ be the output variables of $f_{i}$ and $f_{i}^{*}$, respectively. The circuits DFN on and $\mathrm{DFN}_{\text {off }}$ can be converted to CNFs $C_{\text {on }}$ and $C_{\text {off }}$, respectively, in linear time. In addition, the output of the target function $f$ in $\mathrm{DFN}_{\text {on }}$ is asserted to true, i.e., $y_{0} \equiv 1$; that of $f^{*}$ in $\mathrm{DFN}_{\text {off }}$ is asserted to false, i.e., $y_{0}{ }^{*} \equiv 0$. Furthermore, equality constraints $\left(y_{i} \equiv y_{i}^{*}\right)$ are imposed for $i=1, \ldots, n$. Thereby the entire $\mathrm{CNF} C_{\mathrm{DFN}}$ is

$C_{\text {on }} \wedge C_{\text {off }} \wedge y_{0} \wedge \neg y_{0}{ }^{*} \wedge\left(y_{1} \equiv y_{1}{ }^{*}\right) \wedge \cdots \wedge\left(y_{n} \equiv y_{n}{ }^{*}\right)$,

where $\left(y_{i} \equiv y_{i}^{*}\right)$ is the shorthand for $\left(y_{i} \vee \neg y_{i}^{*}\right) \wedge\left(\neg y_{i} \vee y_{i}^{*}\right)$.

The intuition behind this construct is that formula $C_{\text {on }} \wedge y_{0}$ (respectively $C_{\text {off }} \wedge \neg y_{0}{ }^{*}$ ) imposes the constraint that the valuations over input variables $\left(x_{1}, \ldots, x_{m}\right)$ (respectively $\left(x_{1}{ }^{*}, \ldots\right.$, $\left.x_{m}{ }^{*}\right)$ ) must be the on-set of $f$ (respectively off-set of $\left.f^{*}\right)$. By Proposition 1, we can thus test if $h^{0}$ and $h^{1}$ are disjoint. Precisely speaking, we conclude the following.

Theorem 3. Given a target function $f$ and a set of base functions $g_{i}$ for $i=1, \ldots, n$, a dependency function $h$ exists if and only if the $C N F C_{\mathrm{DFN}}$ of the corresponding DFN is unsatisfiable.

Proof: $(\Rightarrow)$ By Definition $1, f$ can be expressed as $h\left(g_{1}(X), \ldots\right.$, $\left.g_{n}(X)\right)$. Proposition 1 asserts that the onset $h^{1}$ and offset $h^{0}$ of $h$ must be disjoint. Observe that $h^{0}$ and $h^{1}$ are essentially the sets of satisfying assignments of variables $y_{i}$ of $\mathrm{DFN}_{\text {on }}$ and $y_{i}^{*}$ of $\mathrm{DFN}_{\text {off }}$, respectively. Hence CNF $C_{\mathrm{DFN}}$, which is the conjunction of $C_{\mathrm{on}}$ and $C_{\text {off }}$, is unsatisfiable.
$(\Leftarrow)$ If $C_{\mathrm{DFN}}$ is unsatisfiable, there are two cases. For the first case, $C_{\text {on }}$ or $C_{\text {off }}$ is unsatisfiable. It happens only when $f$ is a constant function. $C_{\text {on }}\left(C_{\text {off }}\right)$ is unsatisfiable if and only if $f$ is constant zero (one). In this case, we may express $f$ as a function over any base functions. For the second case, $C_{\mathrm{on}}$ and $C_{\text {off }}$ are both satisfiable. Then unsatisfiable $C_{\mathrm{DFN}}$ implies its clauses of the equality constraints $\left(y_{i} \equiv y_{i}^{*}\right)$ are violated. That is, the sets of images of the onset and offset of $f$ under the base functions are disjoint. By Proposition 1, we know that $h$ must exist.

In the sequel we shall assume a target function is non-constant.

Remark 1. Note that, although DFN is similar to the miter structure used in combinational equivalence checking, the underlying principle is completely different. The DFN construct differs from the miter structure in that: Firstly, the sets of input variables of $\mathrm{DFN}_{\text {on }}$ and $\mathrm{DFN}_{\text {off }}$ are disjoint. Secondly, the output variables of the target functions of $\mathrm{DFN}_{\text {on }}$ and $\mathrm{DFN}_{\text {off }}$ are asserted to true and false, respectively. Thirdly, the equality constraints are imposed only on the corresponding pairs of base functions.

We show how the dependency function can be derived using interpolation provided that the clause set $C_{\mathrm{DFN}}$ is unsatisfiable. To apply Theorem 2, consider partitioning the clause set $C_{\mathrm{DFN}}$ into two subsets $A$ and $B$. We claim the following.

Corollary 1. For unsatisfiable $C_{\mathrm{DFN}}=A \wedge B$ with $A=C_{\mathrm{on}} \wedge y_{0}$ and $B=C_{\text {off }} \wedge \neg y_{0}{ }^{*} \wedge\left(y_{1} \equiv y_{1}{ }^{*}\right) \wedge \cdots \wedge\left(y_{n} \equiv y_{n}{ }^{*}\right)$, the resultant interpolant $A^{\#}$ derived from a refutation proof yields a desired dependency function $h$.

Proof: Observe that the common variables of $A$ and $B$ are $Y=$ $\left(y_{1}, \ldots, y_{n}\right)$, which is desirable for the dependency function. Since $A \wedge B$ is unsatisfiable, by Theorem 2 there exists an interpolant $A^{\#}$ which refers only to $Y$. In addition, conditions $A \Rightarrow A^{\#}$ and $A^{\#} \Rightarrow$ $\neg B$ suggest that the set of valuations over variables $Y$ satisfying $A^{\#}$ must be an over-approximation of $h^{1}(Y)$ and must be disjoint from $h^{0}(Y)$. Hence, $A^{\#}(Y)$ is a valid implementation of the dependency function $h(Y)$ with respect to the underlying target and base functions.

Therefore, as long as a SAT solver can produce an interpolant from a refutation proof, it can be exploited to generate the dependency function. The overall algorithm of the exploration of functional dependency is sketched in Fig. 2.

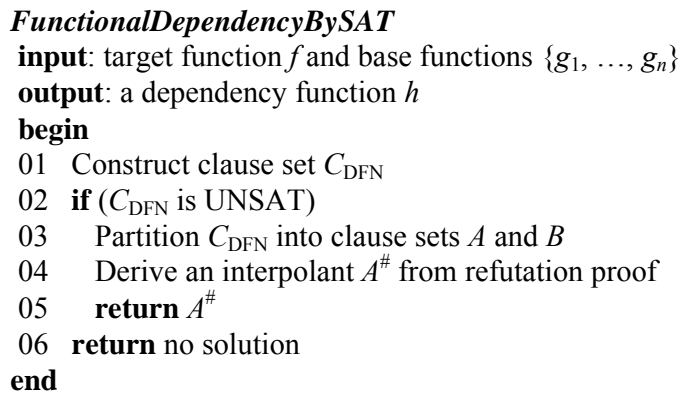

Figure 2. Algorithm: Functional dependency by SAT.

Note that the choice of clause sets $A$ and $B$ is not unique. For instance, letting $A=C_{\text {on }} \wedge y_{0} \wedge\left(y_{1} \equiv y_{1}{ }^{*}\right) \wedge \cdots \wedge\left(y_{n} \equiv y_{n}{ }^{*}\right)$ and $B=$ $C_{\text {off }} \wedge \neg y_{0}{ }^{*}$ is valid as well. In fact, different refutation proofs and different choices of $A$ and $B$ can be exploited to obtain the flexibilities implementing the dependency function. 
Remark 2. One reason that makes our SAT-based approach outperforms BDD-based ones is due to the efficiency in selecting base functions. In our method, we can simply include all candidate base functions rather than iterating over a sequence of carefully selected subsets of the candidate functions. Therefore, our method can detect functional dependency in one run. However, BDD-based methods may require multiple runs.

\subsection{Incremental SAT Solving}

The above discussion assumes the target function is given. However, for a given circuit netlist, there may be many different choices of the target function. Often we need to detect functional dependency for different target functions one at a time. Consider we have explored the functional dependency for target function $f_{0}$ and base functions $\left\{f_{1}, \ldots, f_{n}\right\}$. Suppose now we want to switch the target function to $f_{1}$ and add $f_{0}$ to the base functions. Only slight modification is needed migrating from the original SAT instance, $C_{\mathrm{DFN} 0}$, to the new one, $C_{\mathrm{DFN} 1}$, because the sets of base functions are almost the same. Since the search spaces for the two SAT instances are very similar, incremental SAT solving [21] is helpful in amortizing the computation overhead. We investigate how to incorporate incremental SAT solving in our framework by reusing helpful clauses learned from solving previous SAT instances in subsequent computation.

Since not all previously learned clauses are valid in solving the current SAT instance, invalid clauses need to be disabled. To avoid sophisticated clause removal, we adopt the MiniSat [11] interface, where unit assumptions [11] can be made on a list of literals such that the subsequent SAT solving is restricted to the specified solution subspace and the assumptions are discharged upon return. We introduce auxiliary variables and make unit assumptions on them to enable or disable "dynamic clauses." Let the auxiliary variable controlling clause $c$ be $\alpha_{c}$. We replace $c$ in the original SAT instance with the new clause $\left(\alpha_{c} \Rightarrow c\right)$ such that $c$ is enabled (disabled) when $\alpha_{c}=1(0)$. Because in our case these "dynamic clauses" result from the equality constraints of $C_{\mathrm{DFN}}$, we introduce auxiliary variables $\alpha_{i}$ as the switches of the conditional satisfaction of equality constraints $\left(y_{i} \equiv y_{i}^{*}\right)$. For $\alpha_{i}=1$ (0), equality constraint $y_{i} \equiv y_{i}{ }^{*}$ is turned on (off). For $f_{i}$ to be the target function and the others the base functions, the entire CNF $C_{\text {DFN }}$ of Eq. (1.1) now becomes

$C_{\text {on }} \wedge C_{\text {off }} \wedge y_{i} \wedge \neg y_{i}^{*} \wedge\left(\alpha_{0} \Rightarrow\left(y_{0} \equiv y_{0}{ }^{*}\right)\right) \wedge\left(\alpha_{1} \Rightarrow\left(y_{1} \equiv y_{1}{ }^{*}\right)\right) \wedge \cdots$ $\wedge\left(\alpha_{n} \Rightarrow\left(y_{n} \equiv y_{n}{ }^{*}\right)\right) \wedge \alpha_{0} \wedge \cdots \wedge \alpha_{i-1} \wedge \neg \alpha_{i} \wedge \alpha_{i+1} \wedge \cdots \wedge \alpha_{n}$.

Again to compute interpolants, we partition the above clause set into two subsets $A$ and $B$ with

$A=C_{\text {on }} \wedge y_{i}$ and

$B=C_{\text {off }} \wedge \neg y_{i}^{*} \wedge\left(\alpha_{0} \Rightarrow\left(y_{0} \equiv y_{0}^{*}\right)\right) \wedge\left(\alpha_{1} \Rightarrow\left(y_{1} \equiv y_{1}{ }^{*}\right)\right) \wedge \cdots \wedge\left(\alpha_{n}\right.$ $\left.\Rightarrow\left(y_{n} \equiv y_{n}^{*}\right)\right) \wedge \alpha_{0} \wedge \cdots \wedge \alpha_{i-1} \wedge \neg \alpha_{i} \wedge \alpha_{i+1} \wedge \cdots \wedge \alpha_{n}$.

To check the satisfiability of the above $C_{\mathrm{DFN}}$, the unit clauses $\left\{y_{i}\right.$, $\left.\neg y_{i}^{*}, \alpha_{0}, \cdots, \alpha_{i-1}, \neg \alpha_{i}, \alpha_{i+1}, \cdots, \alpha_{n}\right\}$ will be on the unit assumptions of MiniSat. Effectively, the SAT solving is restricted to the solution subspace with $y_{i}=1, y_{i}^{*}=0, \alpha_{i}=0$ and $\alpha_{j}=1$ for $j \neq i$. If the result is satisfiable, no functional dependency exists under the target function. Otherwise, a conflict clause is returned, which refers only to a subset of the auxiliary variables in addition to the output variables $y_{i}$ and $y_{i}{ }^{*}$ of the target function. It indicates some of the unit assumptions are self-contradicting. Hence there exists a dependency function that depends only on the corresponding base functions. This property gives a quick answer if we are interested only in the input size of the dependency function. Moreover, from this conflict clause we may construct an interpolant under the contradicting solution subspace and thus derive the dependency function.

\subsection{Enumeration of Different Dependency Functions}

For a fixed target function $f$ functionally depending on a set of base functions, it is often the case that the don't care set $\boldsymbol{B}^{n} \backslash\left\{h^{0} \cup h^{1}\right\}$ for the dependency function $h$ is not empty. Hence there is flexibility implementing $h$ differently. Obtaining these don't cares is preferable. However, the capability of SAT solvers is limited in this respect as they tend to find "a" satisfying assignment or "a" refutation proof. A refutation proof uniquely determines an interpolant and, thus, an implementation of the dependency function. To overcome this deficiency, we propose two methods identifying two different types of alternatives of a dependency function implementation: those with the same support variables and those with different ones. For the former, we reorder the resolution sequence of a refutation proof to obtain different interpolants and, thus, different implementations of the dependency function. For instance, the reordering method in [22] is one possibility (where variables local to the clause set $A$ are resolved as early as possible to strengthen interpolants). By proper strengthening and weakening an interpolant, the difference between the strengthened and weakened interpolants provides a subset of the don't cares of $h$. However, practical experience suggests that the so obtained don't care set may not be large. For the latter, we block the SAT solver from searching the same instance and force it to search a new refutation proof with a different set of support variables. This can be done by making proper unit assumptions under the MiniSat interface.

\section{EXPERIMENTAL RESULTS}

The proposed algorithm was implemented in ABC [23] modified to equip with the proof-logging version of MiniSat [11]. All the experiments are conducted on a $3.2 \mathrm{GHz}$ Linux machine with $2 \mathrm{~GB}$ memory. The experiments are designed so as to demonstrate

1. the efficiency and scalability of SAT-based in contrast to BDD-based computation [1],

2. the benefit of our incremental SAT formulation, and

3. the characteristics of the derived dependency functions.

Large circuits from the ISCAS89 and ITC99 benchmark suites are chosen. To have fair comparison with [1], functional dependency among the transition functions of a circuit is explored. Among the transition functions of a given circuit, each of them is specified in turn as the target function and all others as base functions. We then explore the corresponding functional dependency and compute dependency functions if they exist.

Table 1 compares our approach with the prior work [1]. Columns 1 and 2 respectively list the name and the number of nodes of each circuit. The numbers of flip-flops, denoted \#FF, of a circuit and its retimed version (min-period retiming using $\mathrm{ABC}$ [23]) are listed in Columns 3 and 6, respectively. Among the flipflops of an original circuit (respectively a retimed circuit), those whose transition functions possess functional dependency are counted in Columns 4 and 5 (respectively Columns 7 and 8), denoted as \#Dep. In particular, \#Dep-S and \#Dep-B are obtained by the SAT- and BDD-based methods, respectively. In fact, \#Dep-S data are exact and complete. In comparison, the BDDbased method only succeeded in a few circuits and detected only a subset of the dependency over a few support variables. The 
runtime (in seconds) and memory (in Megabytes) usage are shown in the following columns. Note that the reported memory usage includes the underlying system memory whereas the prior work was built on VIS [24] and ours on ABC. Despite the uneven comparison, the scalability of our approach is evident and outperforms the prior work. As plotted in Fig. 3, it is easily seen from the power regression lines that the runtime of our approach scales polynomially with the circuit size.

The strength of incremental SAT solving in accelerating computation is shown in Fig. 4. The $x$-axis and y-axis, respectively, represent the iteration number and the runtime of solving a SAT instance at that particular iteration. The $y$-axis is $\log$-scaled. Five sample circuits of different sizes from Table 1 are plotted for the first 100 iterations. As can be seen in all of the plots, the runtimes of the first iterations are the maximum of among their first 100 iterations. In fact, all the circuits of Table 1 exhibit the same behavior. After the first iteration, the runtimes for SAT solving decrease rapidly and become relatively short and stable within about 10 iterations. This demonstrates the effectiveness for incremental SAT solving.

The experiments tend to suggest that (1) the average runtime for a circuit is linear in the number of its nodes and (2) the solving time for an unsatisfiable SAT instance is often much shorter than that for a satisfiable one. The statistics are plotted in Fig. 5. The $\mathrm{x}$-axis and y-axis, respectively, represent the number of nodes of each circuit and the average runtime of SAT iterations. Both axes are log-scaled. Every circuit in Table 1 is plotted as a spot in Fig. 5. The first tendency can be seen from the two power regression lines indicating highly (positive) correlated data set. The second tendency can be seen from the fact that the line for the retimed circuits is well below that for the original circuits. As evident in Columns 4 and 7 of Table 1, more functional dependency exists for the retimed circuits. Effectively, more unsatisfiable SAT instances are there. It reflects the fact that in our experiments a satisfiable instance usually takes longer time to solve than an unsatisfiable one. It seems contradicting with common sense. However, the tendency can be explained as follows. Because the input sizes of interpolants are mostly very small (to be shown in Fig. 6), it suggests that conflicts can be found locally. Also, incremental SAT solving increases implicativity [25] and thus enhances early conflict detection. Thus, decisions over only a few variables might be enough to draw an unsatisfiable conclusion. In contrast, in a satisfiable case to obtain a single satisfying assignment, decisions must be made over all variables. (Note that it may not be necessarily so for circuit-SAT and other ODCaware CNF solvers, where not all variables are valuated for a satisfying assignment.)

We characterize the derived dependency functions (i.e. interpolants) in terms of their input sizes in Fig. 6, where a single dependency function, if it exists, is derived for each transition function of a given circuit. The $\mathrm{x}$-axis and $\mathrm{y}$-axis indicate the numbers of support variables and of dependency functions, respectively. The y-axis is log-scaled. As can be seen, most of the functions have less than 10 support variables. On the other hand, complex functional dependency can also be detected easily by the SAT-based approach. For instance, in the retimed b18 circuit, a dependency function of input size around 300 is obtained, which is not possible using BDD-based methods. Furthermore, to see if the input variables of an interpolant are indeed irredundant (i.e. support variables), Fig. 7 gives the statistics. (Note that multiple interpolants may locate at the same spot.) For every y-axis index, say $i$, the corresponding number on the right hand side of Fig. 7 indicates the number of interpolants with $i$ spurious variables. As can be seen, no spurious variables appear in most (98.3\%) of the interpolants. Only in few occasions the derived interpolants contain redundancy. On the other hand, Fig. 8 shows the relation between the interpolant size in terms of AIG (And-Inverter Graph) nodes and the number of input variables. The size grows polynomially with respect to the number of input variables, as seen from the two power regression lines. Figures 6, 7 and 8 demonstrate the fact that the derived interpolants are mostly small.

From practical experience in enumerating different dependency functions for a target function, we note that the number of available dependency functions (with different support sets) varies greatly from function to function. A great amount of trivial dependency exists due to the transitivity of dependency. This transitivity results in vast redundant enumeration. How to effectively avoid unnecessary enumeration remains to be done. Nevertheless, if the candidate base functions are specified (e.g. for circuit rewiring), finding a dependency function is easy. On the other hand, we emphasize that the BDD-based method is more effective than the SAT-based one in computing the don't care set for a dependency function. This deficiency of the SAT-based computation is due to the fact that an interpolant (i.e. a dependency function) is with respect to a refutation and contains no don't-care information.

\section{RELATED WORK}

The previous efforts closest to ours are [1] and [4]. Both of them rely on BDD-based computation. In [1] combinational functional dependency was generalized to sequential dependency. In this paper we focus on combinational dependency. In [4], similar to our enumeration for different dependency functions, a BDD-based technique was used. It allows a more implicit enumeration. However, the size of the set of base functions was limited to no more than 16. Other work [18] applied interpolation in the context of SAT-based model checking for approximated image computation.

\section{CONCLUSIONS AND FUTURE WORK}

We have shown that the exploration of functional dependency can be solved by a pure SAT-based formulation. Experimental results demonstrated the great success of the proposed method. The approach is scalable to large designs and discovers much more functional dependency far beyond the capability of prior methods. The success is attributed to several key ingredients including Craig interpolation and incremental SAT solving. We hope that our results may benefit several areas of logic synthesis and formal verification, for example, in finding rewiring and resubstitution candidates for circuit optimization, in identifying redundant registers in RTL synthesis, in reducing state space in formal sequential equivalence checking, etc.

Future work includes integrating our technique in logic synthesis and generalizing it for other applications. (Some preliminary results in synthesizing industrial designs have been demonstrated in [26].) Generalizing our method for sequential dependency [1] is an important subject to pursue. In addition, it would be interesting to explore new applications of Craig interpolation. 
Table 1. SAT- vs. BDD-based Exploration of Functional Dependency.

\begin{tabular}{|c|c|c|c|c|c|c|c|c|c|c|c|c|c|c|c|}
\hline \multirow[b]{2}{*}{ Circuit } & \multirow[b]{2}{*}{ \#Nodes } & \multicolumn{3}{|c|}{ Original } & \multicolumn{3}{|c|}{ Retimed } & \multicolumn{2}{|c|}{ SAT (original) } & \multicolumn{2}{|c|}{ BDD (original) } & \multicolumn{2}{|c|}{ SAT (retimed) } & \multicolumn{2}{|c|}{ BDD (retimed) } \\
\hline & & \#FF. & \#Dep-S & \#Dep-B & \#FF. & \#Dep-S & \#Dep-B & Time & Mem & Time & Mem & Time & Mem & Time & Mem \\
\hline s5378 & 2794 & 179 & 52 & 25 & 398 & 283 & 173 & $1.2 \mathrm{~s}$ & $18 \mathrm{~m}$ & $1.6 \mathrm{~s}$ & $20 \mathrm{~m}$ & $0.6 \mathrm{~s}$ & $18 \mathrm{~m}$ & $7 \mathrm{~s}$ & $51 \mathrm{~m}$ \\
\hline s9234.1 & 5597 & 211 & 46 & $\mathrm{x}$ & 459 & 301 & 201 & $4.1 \mathrm{~s}$ & $19 \mathrm{~m}$ & $\mathrm{x}$ & $\mathrm{x}$ & $1.7 \mathrm{~s}$ & $19 \mathrm{~m}$ & $194.6 \mathrm{~s}$ & $149 m$ \\
\hline s13207.1 & 8022 & 638 & 190 & 136 & 1930 & 802 & $\mathrm{x}$ & $15.6 \mathrm{~s}$ & $22 \mathrm{~m}$ & $31.4 \mathrm{~s}$ & $78 \mathrm{~m}$ & $15.3 \mathrm{~s}$ & $22 \mathrm{~m}$ & $\mathrm{x}$ & $\mathrm{x}$ \\
\hline s15850.1 & 9785 & 534 & 18 & 9 & 907 & 402 & $\mathrm{x}$ & $23.3 \mathrm{~s}$ & $22 \mathrm{~m}$ & $82.6 \mathrm{~s}$ & $94 \mathrm{~m}$ & $7.9 \mathrm{~s}$ & $22 \mathrm{~m}$ & $\mathrm{x}$ & $\mathrm{x}$ \\
\hline s35932 & 16065 & 1728 & 0 & -- & 2026 & 1170 & -- & $176.7 \mathrm{~s}$ & $27 \mathrm{~m}$ & $1117 \mathrm{~s}$ & $164 \mathrm{~m}$ & 78.1 & $27 \mathrm{~m}$ & -- & -- \\
\hline s38417 & 22397 & 1636 & 95 & -- & 5016 & 243 & -- & $270.3 \mathrm{~s}$ & $30 \mathrm{~m}$ & -- & -- & 123.1 & $32 \mathrm{~m}$ & -- & -- \\
\hline s38584 & 19407 & 1452 & 24 & -- & 4350 & 2569 & -- & $166.5 \mathrm{~s}$ & $21 \mathrm{~m}$ & -- & -- & $99.4 \mathrm{~s}$ & $30 \mathrm{~m}$ & $1117 \mathrm{~s}$ & $164 \mathrm{~m}$ \\
\hline b12 & 946 & 121 & 4 & 2 & 170 & 66 & 33 & $0.15 \mathrm{~s}$ & $17 \mathrm{~m}$ & $12.8 \mathrm{~s}$ & $38 \mathrm{~m}$ & $0.13 \mathrm{~s}$ & $17 \mathrm{~m}$ & $2.5 \mathrm{~s}$ & $42 \mathrm{~m}$ \\
\hline b14 & 9847 & 245 & 2 & -- & 992 & 724 & -- & $3.3 \mathrm{~s}$ & $22 \mathrm{~m}$ & -- & -- & $5.2 \mathrm{~s}$ & $22 \mathrm{~m}$ & -- & -- \\
\hline b15 & 8367 & 449 & 0 & -- & 1134 & 793 & -- & $5.8 \mathrm{~s}$ & $22 \mathrm{~m}$ & -- & -- & $5.8 \mathrm{~s}$ & $22 \mathrm{~m}$ & -- & -- \\
\hline b17 & 30777 & 1415 & 0 & -- & 3967 & 2350 & -- & $119.1 \mathrm{~s}$ & $28 \mathrm{~m}$ & -- & -- & $161.7 \mathrm{~s}$ & $42 \mathrm{~m}$ & -- & -- \\
\hline b18 & 111241 & 3320 & 5 & -- & 9254 & 5723 & -- & $1414.9 \mathrm{~s}$ & $100 \mathrm{~m}$ & -- & -- & $2842.6 \mathrm{~s}$ & $100 \mathrm{~m}$ & -- & -- \\
\hline b19 & 224624 & 6642 & 0 & -- & 7164 & 337 & -- & $8184.8 \mathrm{~s}$ & $217 \mathrm{~m}$ & -- & -- & $11040.6 \mathrm{~s}$ & $234 \mathrm{~m}$ & -- & -- \\
\hline b20 & 19682 & 490 & 4 & -- & 1604 & 1167 & -- & $25.7 \mathrm{~s}$ & $28 \mathrm{~m}$ & -- & -- & 36 & $30 \mathrm{~m}$ & -- & -- \\
\hline b21 & 20027 & 490 & 4 & -- & 1950 & 1434 & -- & $24.6 \mathrm{~s}$ & $29 m$ & -- & -- & 36.3 & $31 \mathrm{~m}$ & -- & -- \\
\hline b22 & 29162 & 735 & 6 & -- & 3013 & 2217 & -- & $73.4 \mathrm{~s}$ & $36 \mathrm{~m}$ & -- & -- & 90.6 & $37 \mathrm{~m}$ & -- & -- \\
\hline
\end{tabular}

(“--”: memory usage exceeds $1 \mathrm{~Gb}$. "x": runtime exceeds 12,000 seconds.)

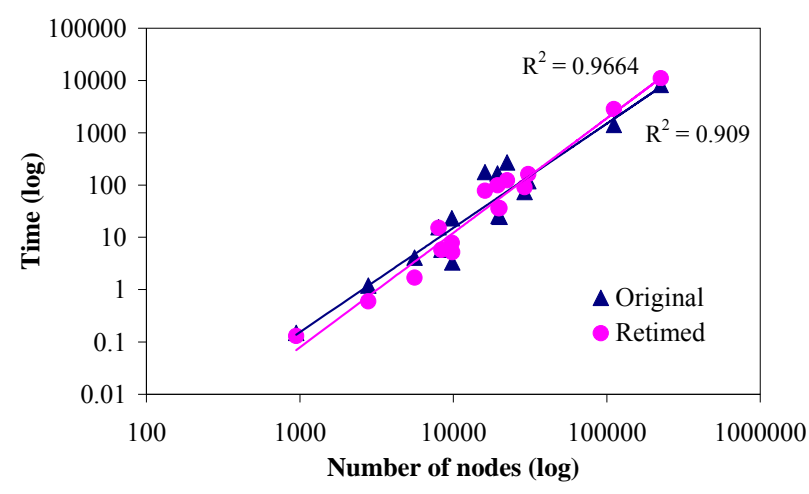

Figure 3. Runtime vs. circuit size.

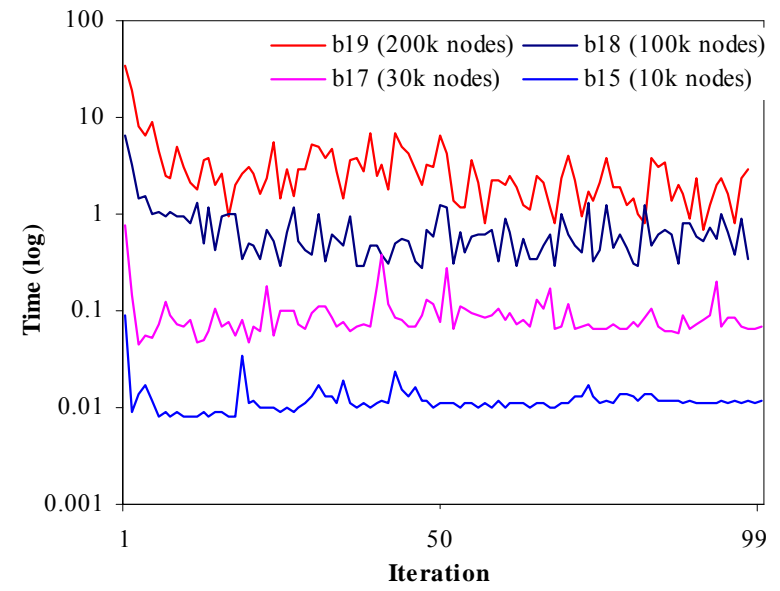

Figure 4. Runtime of the first 100 SAT iterations.

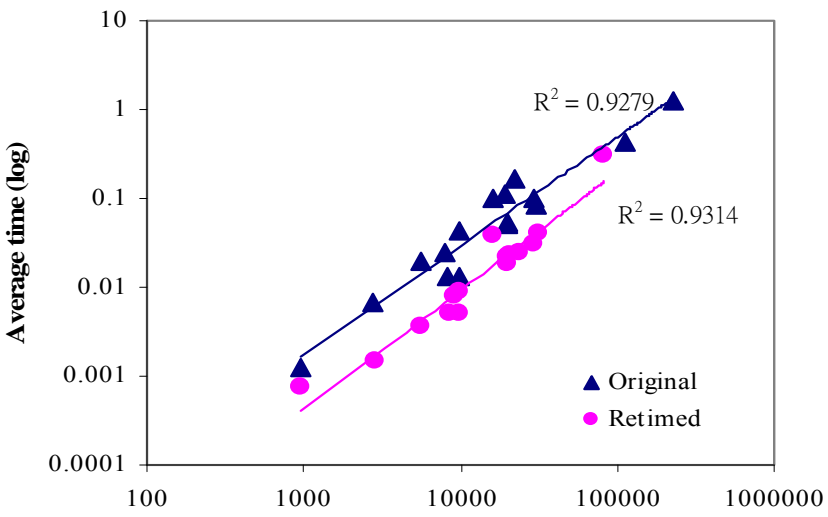

Number of nodes (log)

Figure 5. Average runtime of SAT iterations vs. circuit size.

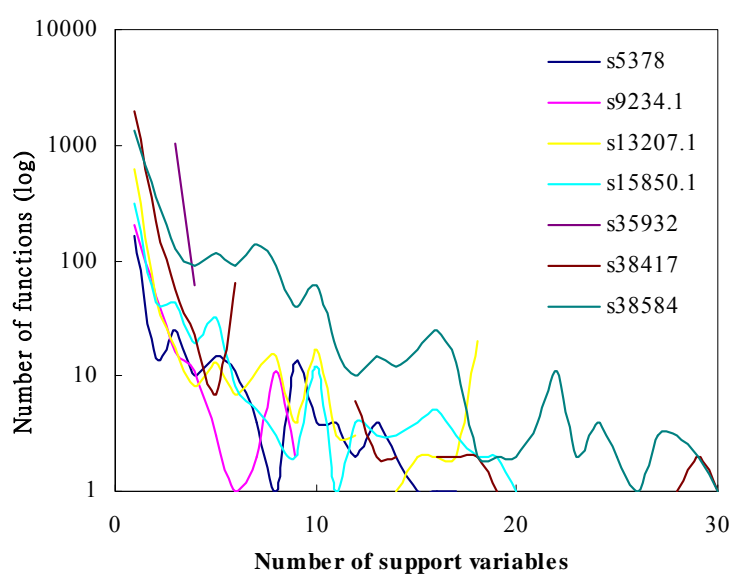

Figure 6. Frequency distribution of different support sizes. 


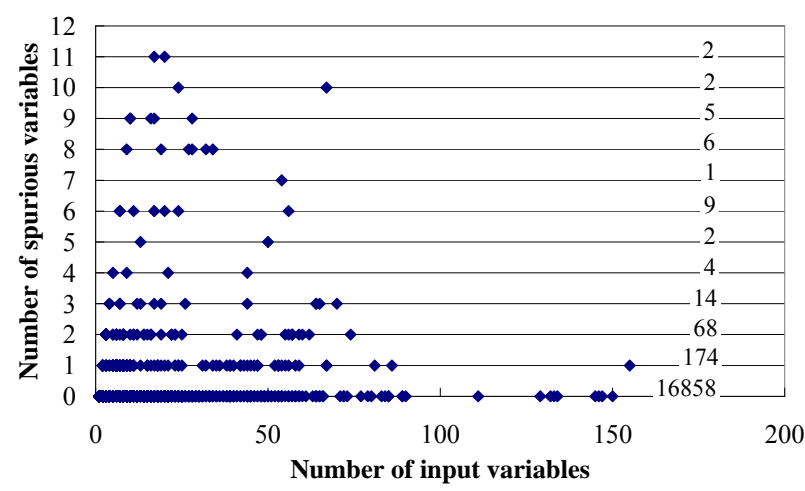

Figure 7. Number of total input variables vs. number of spurious input variables of derived interpolants.

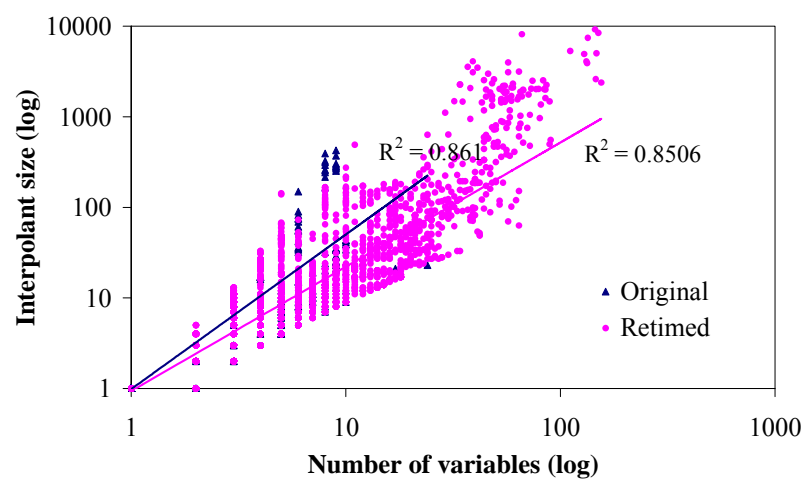

Figure 8. Interpolant size vs. number of input variables.

\section{ACKNOWLEDGMENTS}

The authors would like to thank Robert Brayton for helpful discussions and Ruei-Rung $\mathrm{Li}$ for preparing some of the experimental data. This work was supported in part by NSC grants 95-2221-E-002-432 and 95-2218-E-002-064-MY3. AM was supported by the Intel-custom SRC grant 1444.001 "Innovative Sequential Synthesis and Verification."

\section{REFERENCES}

[1] J.-H. R. Jiang and R. K. Brayton. Functional dependency for verification reduction. In Proc. CAV, pp. 268-280, 2004.

[2] E. Sentovich, H. Toma, and G. Berry. Latch optimization in circuits generated from high-level descriptions. In Proc. ICCAD, pp. 428-435, 1996.

[3] B. Lin and A. R. Newton. Exact redundant state registers removal based on binary decision diagrams. In Proc. Int'l Conf. Very Large Scale Integration, pp. 277-286, 1991.

[4] V. Kravets and P. Kudva. Implicit enumeration of structural changes in circuit optimization. In Proc. DAC, pp. 438-441, 2004.

[5] A. J. Hu and D. L. Dill. Reducing BDD size by exploiting functional dependencies. In Proc. DAC, pp. 266-271, 1993.
[6] C. Berthet, O. Coudert, and J.-C. Madre. New ideas on symbolic manipulations of finite state machines. In Proc. ICCD, pp. 224-227, 1990.

[7] C. A. J. van Eijk and J. A. G. Jess. Exploiting functional dependencies in finite state machine verification. In Proc. European Design \& Test Conf., pp. 9-14, 1996.

[8] E. Gregoire, R. Ostrowski, B. Mazure, and L. Sais. Automatic extraction of functional dependencies. In Proc. SAT, 2004.

[9] R. E. Bryant. Graph-based algorithms for Boolean function manipulation. IEEE Trans. Computers, pp. 677--691, August 1986.

[10] M. Moskewicz, C. Madigan, L. Zhang, and S. Malik. Chaff: Engineering an efficient SAT solver. In Proc. DAC, pp. 530-535, 2001.

[11] N. Eén and N. Sörensson. An extensible SAT-solver. In Proc. SAT, pp. 502-518, 2003.

[12] A. Mishchenko and R. Brayton. SAT-based complete don't-care computation for network optimization. In Proc. DATE, pp. 418-423, 2005.

[13] A. Mishchenko, J. Zhang, S. Sinha, J. Burch, R.K. Brayton, and M. Chrzanowska-Jeske. Using simulation and satisfiability to compute flexibilities in Boolean networks. IEEE Trans. on CAD, vol. 25, no. 5, pp. 742-755, 2006.

[14] W. Craig. Linear reasoning: A new form of the Herbrand-Gentzen theorem. J. Symbolic Logic, 22(3):250-268, 1957.

[15] J. A. Robinson. A machine-oriented logic based on the resolution principle. Journal of the ACM, 12(1):23-41, 1965.

[16] J. Krajicek. Interpolation theorems, lower bounds for proof systems, and independence results for bounded arithmetic. J. Symbolic Logic, 62(2):457-486, June 1997.

[17] P. Pudlak. Lower bounds for resolution and cutting plane proofs and monotone computations. J. Symbolic Logic, 62(3):981-998, September 1997.

[18] K. L. McMillan. Interpolation and SAT-based model checking. In Proc. CAV, pp. 1-13, 2003.

[19] G. Tseitin. On the complexity of derivation in propositional calculus. Studies in Constructive Mathematics and Mathematical Logic, pp. 466-483, 1970.

[20] D. Plaisted and S. Greenbaum. A structure preserving clause form translation. J. Symbolic Computation, vol. 2, pp. 293-304, 1986.

[21] J. Whittemore, J. Kim, and K. Sakallah. SATIRE: A new incremental satisfiability engine. In Proc. DAC, 2001.

[22] R. Jhala and K.L. McMillan, "Interpolant-based transition relation approximation”. Proc. CAV, pp. 39-51, 2005.

[23] Berkeley Logic Synthesis and Verification Group. ABC: A System for Sequential Synthesis and Verification. Release 51205. http://www.eecs.berkeley.edu/ alanmi/abc/

[24] R. K. Brayton, et al. VIS: a system for verification and synthesis. In Proc. CAV, pp. 428--432, 1996.

[25] Y. Novikov and R. Brinkmann. Foundations of Hierarchical SATSolving. In Proc. Int'l Workshop on Boolean Problems, 2004.

[26] A. Mishchenko, R. Brayton, J.-H. R. Jiang, and S. Jang. SAT-based logic optimization and resynthesis. In Proc. IWLS, pp. 358-364, 2007. 